\title{
Moradia e qualidade de vida da pessoa idosa: revisão integrativa
}

\author{
Housing and quality of life of the elderly: integrative review \\ Vivienda y calidad de vida de las personas mayores: revisión integrativa
}

Recebido: 22/01/2021 | Revisado: 24/01/2021 | Aceito: 29/01/2021 | Publicado: 05/02/2021

\author{
Paulo Cordeiro Fontes \\ ORCID: https://orcid.org/0000-0002-4378-4648 \\ Universidade Federal da Paraíba, Brasil \\ E-mail: pcfontes16@gmail.com \\ Edilene Araújo Monteiro \\ ORCID: https://orcid.org/0000-0002-3312-868X \\ Universidade Federal da Paraíba, Brasil \\ E-mail: edilenem06@gmail.com \\ Anderson Belmont Correia de Oliveira \\ ORCID: https://orcid.org/0000-0001-8454-4715 \\ Universidade Federal da Paraíba, Brasil \\ E-mail: andersonbelmont_fisio@hotmail.com \\ Ana Karen Martins de Oliveira \\ ORCID: https://orcid.org/0000-0002-9665-6586 \\ Universidade Federal da Paraíba, Brasil \\ E-mail: ana-psi@hotmail.com \\ Maria Adelaide Silva Paredes Moreira \\ ORCID: https://orcid.org/0000-0001-9460-9172 \\ Universidade Federal da Paraíba, Brasil \\ E-mail: jpadelaide@gmail.com \\ Isabela Albuquerque Passos Farias \\ ORCID: https://orcid.org/0000-0002-3601-1698 \\ Universidade Federal da Paraíba, Brasil \\ E-mail: isabelaapassos@yahoo.com.br \\ Robson Antão de Medeiros \\ ORCID: https://orcid.org/0000-0002-8088-9342 \\ Universidade Federal da Paraíba, Brasil \\ E-mail: robson.antao@gmail.com
}

\begin{abstract}
Resumo
O objetivo desse estudo foi investigar a influência do contexto da moradia na qualidade de vida da pessoa idosa. Trata-se de uma revisão integrativa em que foram selecionadas as seguintes bases de dados e bibliotecas virtuais: PubMED, Web of Science, Scielo, Biblioteca Virtual em Saúde (BVS), Scopus, CINAHL e PsycINFO. Foram identificados 1226 artigos, restando 13 estudos após os critérios de inclusão. Prevaleceu o sexo feminino, com idade acima de 60 anos e residentes no Brasil $(69,2 \%, \mathrm{n}=9)$. Na avaliação da qualidade de vida em idosos, utilizou-se o questionário WHOQOL-bref e/ou WHOQOL-old $(61,5 \%, \mathrm{n}=8)$. Quanto ao contexto da moradia dos idosos evidenciou-se as situações: institucionalizados $(46,1 \%, \mathrm{n}=6$ artigos), sozinho, ou com cuidador. Os resultados indicam que a qualidade de vida das pessoas idosas está relacionada a união de cuidadores e infraestrutura adequada para atender as necessidades peculiares da idade, além de ser fundamental a participação social na manutenção de boas relações de convivência com seus pares.
\end{abstract}

Palavras-chave: Idoso; Qualidade de vida; Habitação para idosos.

\begin{abstract}
The aim of this study was to investigate the influence of the housing context on the quality of life of the elderly. It is an integrative review in which the following databases and virtual libraries were selected: PubMED, Web of Science, Scielo, Virtual Health Library (VHL), Scopus, CINAHL and PsycINFO. The identification of 1226 articles, remaining 13 studies after the inclusion criteria. The female gender prevailed, aged over 60 years and resident in Brazil (69.2\%, $\mathrm{n}=9)$. The WHOQOL-bref and / or WHOQOL-old questionnaire $(61.5 \%, \mathrm{n}=8)$ was used to assess the quality of life of the elderly. As for the context of elderly people's housing, the following situations were observed: institutionalized $(46.1 \%, \mathrm{n}=6$ articles) alone or with caregivers. The results indicate that the quality of life of the elderly is related to the union of caregivers and adequate infrastructure to meet the peculiar needs of their age, in addition to being fundamental to social participation in maintaining good relationships with their peers.
\end{abstract}

Keywords: Aged; Quality of life; Housing for the elderly. 


\section{Resumen}

El objetivo de este estudio fue investigar la influencia del contexto de la vivienda en la calidad de vida de los ancianos. Es una revisión integradora en la que se seleccionaron las siguientes bases de datos y bibliotecas virtuales: PubMED, Web of Science, Scielo, Virtual Health Library (VHL), Scopus, CINAHL y PsycINFO. Se identificaron 1226 artículos, con 13 estudios restantes después de los criterios de inclusión. Prevaleció el género femenino, mayores de 60 años y viviendo en Brasil $(69,2 \%, \mathrm{n}=9)$. Para evaluar la calidad de vida en los ancianos, se utilizó el cuestionario WHOQOL-bref y / o WHOQOL-old $(61,5 \%, \mathrm{n}=8)$. En cuanto al contexto de la vivienda de los ancianos, se resaltaron las situaciones: institucionalizadas $(46,1 \%, \mathrm{n}=6$ artículos), solas o con un cuidador. Los resultados indican que la calidad de vida de las personas mayores está relacionada con la unión de los cuidadores y la infraestructura adecuada para atender las necesidades peculiares de su edad, además de ser fundamental para la participación social en el mantenimiento de buenas relaciones con sus pares.

Palabras clave: Anciano; Calidad de vida; Viviendas para anciano.

\section{Introdução}

A qualidade de vida em pessoas idosas envolve um contexto de habitação digna como facilitadora de uma melhor convivência e bem-estar, situações específicas adaptadas às necessidades, além de meio de transportes. Sobretudo, é importante levar em consideração os anseios, expectativas da pessoa idosa como sujeito de decisões e autonomia, respeitando a sua individualidade e o contexto social no qual ela encontra-se inserida (Oliveira et al., 2016).

$\mathrm{Na}$ perspectiva do contexto social, a família é a base, o suporte onde os laços afetivos são enriquecedores e fortalecedores nas relações de trocas que acontecem num espaço ou ambiente propiciador da convivência familiar e comunitária (Dias, Carvalho \& Araújo, 2013). Na ausência de suporte familiar, o fator institucional deve ter um maior reconhecimento e importância, principalmente diante da transição populacional e demográfica. Em uma perspectiva de assistência adequada à pessoa idosa, assim como os idosos em cuidados domiciliares, é necessário que o vínculo familiar esteja aliado a desenvolver estímulos ao estilo de vida saudável, favorecer a realização e satisfação quanto à qualidade de vida, buscando atender as demandas da população que envelhece (Oliveira et al., 2016). O condomínio para pessoas idosas proporciona o direito de uma habitação digna ao prezar pela manutenção da sua qualidade de vida (Teston \& Marcon, 2015). Ao considerar que a avaliação do indivíduo com relação à qualidade de vida pode variar de acordo com o contexto de moradia, tempo e prioridades ao longo da vida, justifica-se investigar a influência da convivência no contexto da moradia na qualidade de vida em pessoas idosas. Nesses termos, o objetivo desse trabalho foi investigar a influência do contexto da moradia na qualidade de vida da pessoa idosa.

\section{Metodologia}

A revisão integrativa foi conduzida no presente estudo por ser ferramenta ímpar na área da saúde por permitir a busca, a avaliação crítica e a síntese das evidências disponíveis do tema investigado para compreensão de um fenômeno ou um problema, obtendo-se resultado sólido para Prática Baseada em Evidências (Broome, 2000; Souza, Silva \& Carvalho, 2010; Pereira et al., 2018).

O presente estudo seguiu com elaboração da seguinte pergunta norteadora: "Qual a influência da moradia na qualidade de vida do idoso?’. A etapa seguinte foi a busca ou amostragem na literatura. Para isso, foram selecionadas as seguintes bases de dados e bibliotecas virtuais para busca: PubMED, Web of Science, Scielo, Biblioteca Virtual em Saúde (BVS), Scopus, Cinahl E Psycinfo. Os descritores combinados foram: qualidade de vida (quality of life), habitação para idoso (housing for the elderly; Homes for the Aged), idoso (aged, elderly).

Após a busca inicial, foram aplicados os critérios de inclusão para a seleção da amostra constituída por artigos científicos com texto na íntegra sobre qualidade de vida de idosos (60 anos ou mais) contemplando a temática da convivência no contexto da habitação ou moradia; artigos nos idiomas português, inglês e/ ou espanhol publicados no período de janeiro de 
2013 a dezembro de 2020.

Da seleção, foram excluídas monografias, tese e dissertações, artigos que não utilizaram instrumento de avaliação da qualidade de vida, artigos relacionados à pesquisa metodológica como criação e/ou validação de instrumentos de qualidade de vida, revisões sistemáticas e aqueles que não envolveram seres humanos.

\section{Resultados e Discussão}

O cruzamento dos descritores permitiu a identificação de 1226 artigos, sendo 123 da PubMED, 232 Web of Science, 18 Scielo, 55 BVS, 595 Scopus, 111 Cinahl e 92 Psyc INFO, restando 13 estudos após aplicação dos critérios de inclusão.

Dos 13 artigos, em 09 (69,2\%), o sexo feminino foi o mais frequente, com idade acima de 60 anos e residentes no Brasil (Tabela 1). A predominância do sexo feminino pode ser justificada pelo aumento da expectativa de vida feminina nas últimas décadas (Lima, Teston, \& Marcon, 2014). Além disso a maioria dos estudos foram realizados na zona urbana em que se observa uma maior prevalência, provavelmente relacionada a migração das mulheres do campo para cidade em busca de serviços de saúde (Tavares et al., 2015).

Em relação ao tipo de instrumento de avaliação da qualidade de vida em pessoas idosas, observou-se que 8 (61,5\%) artigos utilizaram o questionário WHOQOL-bref e/ou WHOQOL-old (Tabela 1).

Tabela 1 - Distribuição dos artigos segundo autoria, título, tipo de estudo e caracterização da amostra.

\begin{tabular}{|c|c|c|c|c|c|c|}
\hline Autor (ano) & Periódico & Amostra & Sexo & Idade & $\begin{array}{c}\text { Instrumento de } \\
\text { avaliação de QV }\end{array}$ & Local \\
\hline $\begin{array}{l}\text { Dias, Carvalho } \\
\text { \& Araújo } \\
\text { (2013) }\end{array}$ & $\begin{array}{l}\text { Revista } \\
\text { Brasileira de } \\
\text { Geriatria e } \\
\text { Gerontologia }\end{array}$ & $\begin{array}{c}51 \text { idosos }(\geq 60 \\
\text { anos })\end{array}$ & $56,8 \%$ feminino & $\begin{array}{l}68,9 \pm 4,27 \\
\text { anos }\end{array}$ & $\begin{array}{l}\text { WHOQOL-bref e } \\
\text { WHOQOL-old }\end{array}$ & Brasil \\
\hline $\begin{array}{c}\text { Gonçalves et.al } \\
\text { (2013) }\end{array}$ & $\begin{array}{l}\text { Revista } \\
\text { Brasileira de } \\
\text { Geriatria e } \\
\text { Gerontologia }\end{array}$ & 100 idosos & $63 \%$ feminino & $\begin{array}{l}\text { Média de } 84 \\
\text { anos. }\end{array}$ & $\begin{array}{l}\text { WHOQOL-bref e } \\
\text { WHOQOL-old }\end{array}$ & Brasil \\
\hline $\begin{array}{l}\text { Orrell et al. } \\
\text { (2013) }\end{array}$ & $\begin{array}{l}\text { Health \& } \\
\text { Place }\end{array}$ & $\begin{array}{l}163 \text { pessoas } \\
\text { idosas }\end{array}$ & $65 \%$ feminino & $\begin{array}{c}\text { Mediana } 80 \\
\text { anos feminino } \\
\text { - Mediana } 79 \\
\text { anos }\end{array}$ & SEIQoL-DW ${ }^{\text {a }}$ & $\begin{array}{l}\text { Reino } \\
\text { Unido }\end{array}$ \\
\hline $\begin{array}{l}\text { Ratcliffe et al. } \\
\text { (2013) }\end{array}$ & $\begin{array}{l}\text { Health and } \\
\text { social care in } \\
\text { the community }\end{array}$ & $\begin{array}{l}671 \text { pessoas } \\
\text { idosas sem } \\
\text { cuidadores e } \\
115 \text { pessoas } \\
\text { idosas com } \\
\text { cuidadores } \\
\text { (parente, amigo } \\
\text { ou esposo). }\end{array}$ & $\begin{array}{l}\text { Com cuidador: } 67 \% \\
\text { feminino; Sem } \\
\text { cuidador: } 61 \% \\
\text { feminino }\end{array}$ & $\begin{array}{c}\text { Maiores de } 65 \\
\text { anos. }\end{array}$ & ICECAP-O $^{b}$ & $\begin{array}{c}\text { Sul da } \\
\text { Austrália }\end{array}$ \\
\hline $\begin{array}{l}\text { Khoury \& Sá- } \\
\text { Neves (2014). }\end{array}$ & $\begin{array}{l}\text { Revista } \\
\text { Brasileira de } \\
\text { Geriatria e } \\
\text { Gerontologia }\end{array}$ & $\begin{array}{l}66 \text { pessoas } \\
\text { idosas }\end{array}$ & $69,2 \%$ feminino & $\begin{array}{c}\text { Média de } 74,9 \\
\pm 8,89\end{array}$ & WHOQOL-old & Brasil \\
\hline $\begin{array}{l}\text { Lima, Teston \& } \\
\text { Marcon (2014) }\end{array}$ & $\begin{array}{l}\text { Saúde (Santa } \\
\text { Maria) }\end{array}$ & $\begin{array}{l}50 \text { pessoas } \\
\text { idosas }\end{array}$ & $62 \%$ feminino & $\begin{array}{c}\text { Entre } 60 \text { a } 79 \\
\text { anos }(86 \%)\end{array}$ & $\begin{array}{l}\text { WHOQOL-bref e } \\
\text { WHOQOL-old }\end{array}$ & Brasil \\
\hline $\begin{array}{c}\text { Teston \& } \\
\text { Marcon (2014) }\end{array}$ & $\begin{array}{l}\text { Revista } \\
\text { Gaúcha de } \\
\text { Enfermagem }\end{array}$ & $\begin{array}{l}20 \text { pessoas } \\
\text { idosas }\end{array}$ & $60 \%$ feminino & $\begin{array}{l}\text { Média de } 72 \\
\text { anos }\end{array}$ & $\begin{array}{l}\text { Questionário semi } \\
\text { estruturado com três } \\
\text { questões norteadoras }^{\mathrm{c}}\end{array}$ & Brasil \\
\hline
\end{tabular}


Research, Society and Development, v. 10, n. 2, e8910212277, 2021

(CC BY 4.0) | ISSN 2525-3409 | DOI: http://dx.doi.org/10.33448/rsd-v10i2.12277

\begin{tabular}{|c|c|c|c|c|c|c|}
\hline $\begin{array}{l}\text { Brandão \& Zatt } \\
\text { (2015) }\end{array}$ & Aletheia & 8 idosos & $50 \%$ feminino & N.I. & $\begin{array}{l}\text { Entrevista } \\
\text { semiestruturada } \\
\text { audiogravada. }\end{array}$ & Brasil \\
\hline $\begin{array}{l}\text { Oliveira et.al. } \\
\quad(2015)\end{array}$ & $\begin{array}{c}\text { Revista } \\
\text { Faculdade } \\
\text { Montes Belos }\end{array}$ & $\begin{array}{c}17 \text { idosos } \\
\text { residentes em } \\
\text { uma ILPI e } 17 \\
\text { idosos em CDM }\end{array}$ & $\begin{array}{c}\text { ILPI: } 13,52 \% \\
\text { feminino e } 52,96 \% \\
\text { masculino; CDM: } \\
\text { 13,52\% feminino e } \\
52,96 \% \text { masculino }\end{array}$ & $\begin{array}{c}\text { Faixa etária } \\
\text { de } 60 \text { a } 89 \\
\text { anos }\end{array}$ & WHOQOL-bref & Brasil \\
\hline $\begin{array}{l}\text { Tavares et al. } \\
\text { (2015) }\end{array}$ & $\begin{array}{c}\text { Revista Baiana } \\
\text { de } \\
\text { Enfermagem,S } \\
\text { alvador }\end{array}$ & $\begin{array}{l}850 \text { idosos na } \\
\text { zona rural e } \\
1942 \text { idosos na } \\
\text { urbana. }\end{array}$ & $\begin{array}{l}62,5 \% \text { feminino na } \\
\text { zona urbana e } \\
52,8 \% \text { masculino na } \\
\text { zona rural. }\end{array}$ & $\begin{array}{c}\text { Média de } 71, \\
38 \pm 7,54 \text { na } \\
\text { zona urbana e } \\
68,73 \pm 7,07 \\
\text { na zona rural }\end{array}$ & $\begin{array}{l}\text { WHOQOL-bref } \\
\text { WHOQOL-old }\end{array}$ & Brasil \\
\hline $\begin{array}{c}\text { Teston \& } \\
\text { Marcon (2015) }\end{array}$ & $\begin{array}{l}\text { Investigación } \\
\text { y Educación } \\
\text { en Enfermería }\end{array}$ & $\begin{array}{l}\mathrm{G} 1: 50 \\
\text { residentes em } \\
\text { uma instituição } \\
\text { geriátrica e G2: } \\
173 \text { pessoas } \\
\text { idosas } \\
\text { residentes em } \\
\text { suas casas. }\end{array}$ & $\begin{array}{c}\text { Feminino (G1: } 62 \% \\
\text { e G2: } 69 \%\end{array}$ & $\begin{array}{c}\text { Superior a } 60 \\
\text { anos }\end{array}$ & $\begin{array}{l}\text { WHOQOL-bref } \\
\text { WHOQOL-old }\end{array}$ & Brasil \\
\hline $\begin{array}{c}\text { Čanković et al. } \\
\text { (2016) }\end{array}$ & $\begin{array}{l}\text { Vojnosanitetsk } \\
\text { i Pregled }\end{array}$ & $\begin{array}{l}200 \text { pessoas } \\
\text { idosas }\end{array}$ & $69,8 \%$ feminino & 79,2 anos & WHOQOL-bref & $\begin{array}{l}\text { República } \\
\text { da Sérvia }\end{array}$ \\
\hline $\begin{array}{l}\text { Henning-Smith } \\
\text { (2016) }\end{array}$ & $\begin{array}{c}\text { Journal } \\
\text { Applied } \\
\text { Gerontology }\end{array}$ & $\begin{array}{l}36 \% \text { viviam } \\
\text { sozinhos; } 50 \% \\
\text { viviam com } \\
\text { cônjuge ou } \\
\text { parceiro; } 5 \% \\
\text { viviam com } \\
\text { cônjuge/ } \\
\text { parceiros e } \\
\text { outros; } 10 \% \\
\text { viviam com } \\
\text { outros }\end{array}$ & - & $\begin{array}{c}\text { Acima de } 65 \\
\text { anos }\end{array}$ & $\begin{array}{l}\text { Pergunta: "Em geral, } \\
\text { você diria que sua } \\
\text { qualidade de vida é..." }\end{array}$ & $\begin{array}{l}\text { Estados } \\
\text { Unidos }\end{array}$ \\
\hline
\end{tabular}

${ }^{1}$ Schedule for the Evaluation of the Individual Quality of Life-Direct Weighting

${ }^{2}$ Index of Capability

${ }^{3}$ O que significa ter QV para o senhor?; 2. Como o senhor avalia suas condições de vida antes e depois de se mudar para

o condomínio?; 3. Houve alguma mudança em suas condições de vida depois de ter se mudado para cá?

Fonte: Autores.

A Tabela 2 evidencia os objetivos, o contexto da moradia das pessoas idosas e resultados apresentados. No que tange ao contexto de moradia, evidenciou-se algumas situações: 6 artigos que abordavam institucionalizados (46,1\%), sozinho, com cuidador formal e informal. 
Tabela 2 - Distribuição dos artigos de acordo com os objetivos, instrumento de avaliação da Qualidade de vida e conclusões.

\begin{tabular}{llll}
\hline Autor (ano) & Objetivos & Contexto da moradia & Resultados/ Conclusões \\
\hline $\begin{array}{l}\text { Dias, Carvalho \& } \\
\text { Araújo (2013) }\end{array}$ & $\begin{array}{l}\text { Comparar a percepção subjetiva de } \\
\text { qualidade de vida e bem-estar de } \\
\text { idosos que vivem sozinhos, com a } \\
\text { família e institucionalizados, por }\end{array}$ & $\begin{array}{l}\text { Sozinhos, com a } \\
\text { família e }\end{array}$ & $\begin{array}{l}\text { A percepção de qualidade de vida foi satisfatória e } \\
\text { similar entre os grupos. Destacando-se que os }\end{array}$ \\
& meio dos instrumentos WHodos & $\begin{array}{l}\text { idosos institucionalizados apresentaram maior grau } \\
\text { de satisfação em relação ao domínio físico. }\end{array}$ \\
\end{tabular}

BREF e WHOQOL-OLD.

\begin{tabular}{ll}
\hline Gonçalves et al. & Avaliar a dinâmica da família, a \\
qualidade e o estilo de vida de \\
idosos da quarta idade e de seus \\
familiares cuidadores.
\end{tabular}

Com a Família

Com a Familia

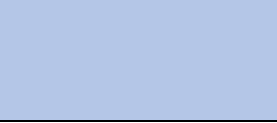

$\begin{array}{lll}\begin{array}{l}\text { Orrell et al. } \\ (2013)\end{array} & \text { Avaliar a qualidade de vida de } \\ & \text { pessoas idosas residentes no } \\ & \begin{array}{l}\text { Esquemas de habilitação de } \\ \text { cuidados extras. }\end{array}\end{array}$

$\begin{array}{lr}\text { Esquemas } & \text { de } \\ \text { habilitação } & \text { de } \\ \text { cuidados extras } & \text { (flats } \\ \text { e bangalôs } & \text { próprios } \\ \text { das pessoas } & \text { idosas } \\ \text { com uma variedade de } \\ \text { instalações e } & \text { apoio } \\ \text { projetados } & \text { para } \\ \text { atender às } & \text { suas } \\ \text { necessidades) } & \end{array}$

\begin{tabular}{lll}
\hline $\begin{array}{l}\text { Ratcliffe et al. } \\
(2013)\end{array}$ & $\begin{array}{l}\text { Avaliar a qualidade de vida de uma } \\
\text { amostra de pessoas idosas } \\
\text { representativa da antiga população } \\
\text { da Austrália do Sul de acordo com } \\
\end{array}$ \\
& o status de cuidador.
\end{tabular}

\section{Khoury \& Sá- Investigar}

\begin{tabular}{ll}
\hline Lima, Teston \& & $\begin{array}{l}\text { Comparar a qualidade de vida de } \\
\text { Marcon (2014) } \\
\text { idosos que residem sozinhos com a } \\
\text { de idosos que moram com } \\
\text { companheiro }\end{array}$
\end{tabular}

Com cuidador (parente, amigo ou esposo) e sem cuidador

As pessoas idosas com cuidadores tiveram associação com alta qualidade de vida quando comparados aos não cuidados. Mesmos que os cuidadores sejam informais.

A percepção de qualidade de vida foi satisfatória. Com o avançar da idade há comprometimento da faceta participação social, além de uma tendência a diminuição dos escores da qualidade de vida. A dinâmica de funcionalidade familiar estava comprometida observando alta disfuncionalidade, pelas exigências de cuidado requerida pela pessoa idosa com afecções crônicas e outros agravos

Um design de construção de boa qualidade refletiu em uma boa qualidade de vida com alto nível de satisfação.
Neves (2014). percepções de controle - primário e secundário - e qualidade de vida em uma amostra de

idosos em Belém-PA, comparandose institucionalizados com residentes na comunidade,

buscando-se associação entre estas variáveis nesses contextos de existência.

\begin{abstract}
Institucionalizados e Viver em comunidade favorece a qualidade de vida não

institucionalizados em todas as facetas com exceção da dimensão "morte e morrer". Qualidade de vida de nível moderado entre institucionalizados.
\end{abstract}

Residentes sozinhos e Pessoas idosas que moram com companheiro casais apresentam melhores escores de QV quando comparados aos idosos que residem só, em relação ao domínio psicológico e faceta intimidade. Escores satisfatórios para os dois grupos foram observados, referentes às relações e participações sociais.

Teston \& Marcon Conhecer como os residentes de um (2014) "condomínio do idoso" percebem a qualidade e condições.

de vida nessa nova modalidade habitacional
Residentes de um A nova modalidade de "condomínio do idoso". qualidade de vida - a estrutura

física, o convívio social entre os moradores,e as condições relacionadas a segurança e a privacidade.O convívio social foi destacado como fundamental para que a pessoa viva melhor. $\mathrm{O}$ espaço de convívio e interação entre as pessoas idosas permite a construção de espaços simbólicos 


\begin{tabular}{|c|c|c|c|}
\hline & & & de partilha dos significados. \\
\hline $\begin{array}{l}\text { Brandão \& Zatt } \\
(2015)\end{array}$ & $\begin{array}{l}\text { Investigar a percepção sobre } \\
\text { qualidade de vida sob a perspectiva } \\
\text { de idosos institucionalizados. }\end{array}$ & $\begin{array}{l}\text { Idosos } \\
\text { institucionalizados. }\end{array}$ & $\begin{array}{l}\text { Novas vivências e boas relações são contribuintes } \\
\text { para uma boa saúde ao proporcionar estímulos de } \\
\text { bem-estar. }\end{array}$ \\
\hline $\begin{array}{l}\text { Oliveira et.al. } \\
(2015)\end{array}$ & $\begin{array}{l}\text { Comparar a qualidade de vida (QV) } \\
\text { dos idosos que vivem em ILPI's, e } \\
\text { aqueles que vivem em cuidados } \\
\text { domiciliares (CDM). }\end{array}$ & ILPI e CDM & $\begin{array}{l}\text { O grau de satisfação dos ILPI e CDM variou entre } \\
\text { regular e bom. Os idosos institucionalizados } \\
\text { demonstraram uma insatisfação quanto a sua } \\
\text { qualidade de vida nos domínios físicos e relações } \\
\text { sociais, enquanto os idosos em CDM apresentaram } \\
\text { níveis baixos de satisfação nos domínios } \\
\text { psicológicos e meio ambiente. }\end{array}$ \\
\hline $\begin{array}{l}\text { Tavares et al. } \\
(2015)\end{array}$ & $\begin{array}{l}\text { Comparar características } \\
\text { sociodemográficas e verificar } \\
\text { preditores associados aos menores } \\
\text { escores de qualidade de vida (QV) } \\
\text { de idosos, segundo local de } \\
\text { moradia. Inquérito transversal } \\
\text { realizado nas zonas rural e urbana, } \\
\text { utilizando instrumentos } \\
\text { estruturados e validados. }\end{array}$ & $\begin{array}{l}\text { Idosos na zona rural e } \\
\text { zona urbana. }\end{array}$ & $\begin{array}{l}\text { A ausência de companheiro foi preditor de menor } \\
\text { escore para ambas localidades. }\end{array}$ \\
\hline $\begin{array}{l}\text { Teston \& Marcon } \\
(2015)\end{array}$ & $\begin{array}{l}\text { Comparar a qualidade de vida de } \\
\text { idosos residentes em uma } \\
\text { instituição geriátrica contra aqueles } \\
\text { que residem em suas casas. }\end{array}$ & $\begin{array}{l}\text { Residentes em uma } \\
\text { instituição geriátrica } \\
\text { contra aqueles que } \\
\text { residem em suas } \\
\text { casas. }\end{array}$ & $\begin{array}{l}\text { A qualidade de vida não apresentou associação } \\
\text { com local de moradia. }\end{array}$ \\
\hline $\begin{array}{l}\text { Čanković et al. } \\
(2016)\end{array}$ & $\begin{array}{l}\text { Avaliar a relação entre dados } \\
\text { sociodemográficos e qualidade de } \\
\text { vida. }\end{array}$ & ILP & $\begin{array}{l}\text { Não houve associação entre dados } \\
\text { sociodemográficos e qualidade de vida, exceto } \\
\text { item doença. }\end{array}$ \\
\hline $\begin{array}{l}\text { Henning-Smith } \\
\text { (2016) }\end{array}$ & $\begin{array}{l}\text { Avaliar qualidade devida de } \\
\text { pessoas idosas em diferentes } \\
\text { contextos de convivência. }\end{array}$ & $\begin{array}{l}\text { Sozinhos; } \\
\text { cônjuge ou } \\
\text { Com } \\
\text { parceiro; } \\
\text { parceiros e e outros } \\
\text { Com outros }\end{array}$ & $\begin{array}{l}\text { Não foi avaliado diferença entre qualidade de vida } \\
\text { entre conviver sozinho e demais tipos de arranjos. }\end{array}$ \\
\hline
\end{tabular}

Fonte: Autores.

A rotina de vida das pessoas idosas é diferente de acordo com contexto da moradia e isso reflete diretamente na qualidade de vida, como observado por Khoury e Sá-Neves (2014) que afirmaram que a institucionalização é um fator negativo para a qualidade de vida. $\mathrm{O}$ item da autonomia pela pessoa idosa revelar nesse ambiente uma menor capacidade para produzir resultados, enquanto morar em comunidade tem uma maior possibilidade de comandar sua rotina diária. Entretanto, outro estudo (Dias, Carvalho \& Araújo, 2013) afirmou que a percepção de qualidade de vida foi satisfatória e similar entre as pessoas idosas que moram sozinhas, com a família e os institucionalizados. Assim, entende-se o ambiente físico no qual a pessoa idosa está inserida, como influenciador direto na dependência desse indivíduo (Dias, Carvalho \& Araújo, 2013).

No contexto da autonomia e moradia, as pessoas idosas institucionalizadas possuem menos atividades de vida diária, como arrumar a casa, cozinhar, fazer compras e pagar contas, o que pode, também, influenciar na percepção em relação ao domínio físico.2 Desta avaliação corrobora Oliveira et al. (2016) em relação a insatisfação quanto à qualidade devida no domínio físico institucionalizado.

Independente do contexto da moradia, deve-se buscar o planejamento de estratégias para preservação da autonomia da pessoa idosa para sua qualidade de vida. Assim, o desenvolvimento de atividades direcionadas à manutenção, proporcionando o cuidado de si, educação e saúde além da estimular a prática de exercício físico são fatores preponderantes para o bem-estar (Teston; Marcon,2014).

Outro fator relevante na qualidade de vida é o convívio social por contribuir para o envelhecimento saudável (Dias, 
Carvalho, Araújo \& 2013; Lima, Teston \& Marcon, 2014; Brandão \& Zatt, 2015). As relações sociais parecem estarem desfavorecidas no contexto da institucionalização (Oliveira et al., 2016). Para favorecer o convívio social, um design de construção de boa qualidade propicia a interação social, permitindo a construção de espaços de partilha dos significados e construção de novas vivencias (Teston \& Marcon, 2014; Orrell et al., 2013).

O convívio social da pessoa idosa sob cuidados domiciliares pode ser limitado, com influência direta na qualidade de vida relacionada ao domínio psicológico (Oliveira et al., 2016). Em qualquer espaço físico em que a pessoa idosa esteja inserida deve-se estimular o convívio social e a autonomia, considerando que o isolamento social é fator de declínio da saúde física e mental (Oliveira et al., 2016).

A presença do cuidador influencia positivamente na qualidade de vida da pessoa idosa dependente por favorecer a interação social e o cuidado em saúde (Lima, Teston \& Marcon, 2014; Ratcliffe et al., 2013). Como destacado por outro estudo (Tavares et al., 2015), a ausência de companheiro é preditor para menor percepção da qualidade de vida. Entretanto HenningSmith (2016) não verificou diferença entre qualidade de vida entre conviver sozinho e demais tipos de arranjos. Essa informação corrobora com outro trabalho (Gonçalves et al., 2013) que verifica a percepção de qualidade de vida satisfatória entre residentes com a família.

\section{Considerações Finais}

O presente estudo evidenciou a escassez de estudos transversais e longitudinais na temática proposta, de maneira a realmente identificar como os espaços de permanência da pessoa idosa influenciam na sua qualidade de vida, respeitando o seu olhar, de maneira a fomentar estratégias para o seu futuro.

Os resultados indicaram que a qualidade de vida das pessoas idosas está relacionada a união de cuidadores e infraestrutura adequada para atender as necessidades peculiares da idade, além de ser fundamental a participação social na manutenção de boas relações de convivência com seus pares. Além disso, reitera-se que um fator relevante é a falta de recursos familiares para responder aos cuidados da pessoa idosa, apontando a importância de políticas públicas direcionadas para proporcionar uma qualidade de vida, independente do contexto de moradia.

\section{Referências}

Broome, M. E. (2000). Integrative literature reviews for the development of concepts. In: Rodgers, B. L., Knafl, K. A, editors. Concept development in nursing: foundations, techniques and applications. Philadelphia: W.B Saunders Company.

Brandão, V. C., \& Zatt, G. B. (2015). Percepção de idosos, moradores de uma instituição de longa permanência de um município do interior do Rio Grande do Sul, sobre qualidade de vida. Aletheia, (46).

Dias, D. D. S. G., Carvalho, C. D. S., \& Araújo, C. V. D. (2013). Comparação da percepção subjetiva de qualidade de vida e bem-estar de idosos que vivem sozinhos, com a família e institucionalizados. Revista brasileira de Geriatria e Gerontologia, 16(1), 127-138.

Gonçalves, L. T. H., Leite, M. T., Hildebrandt, L. M., Bisogno, S. C., Biasuz, S., \& Falcade, B. L. (2013). Convívio e cuidado familiar na quarta idade: qualidade de vida de idosos e seus cuidadores. Revista Brasileira de Geriatria e Gerontologia, 16(2), 315-325.

Henning-Smith, C. (2016). Quality of life and psychological distress among older adults: The role of living arrangements. Journal of Applied Gerontology, $35(1), 39-61$.

Khoury, H. T. T., \& Sá-Neves, Â. C. (2014). Percepção de controle e qualidade de vida: comparação entre idosos institucionalizados e não institucionalizados. Revista Brasileira de Geriatria e Gerontologia, 17(3), 553-565.

Lima, J., Teston, E. F., \& Marcon, S. S. (2014). Qualidade de vida de residentes em condomínio exclusivo para idosos. Saúde (Santa Maria), 73-80.

Oliveira, D. A., Siqueira, R. C., Nogueira, M. S., Alves, A. G., de Brito, F. A. V., \& de Castro Villar, A. (2016). Estudo comparativo da qualidade de vida de idosos institucionalizados e idosos em cuidado domiciliar. Revista Eletrônica Faculdade Montes Belos, 8(3).

Orrell, A., McKee, K., Torrington, J., Barnes, S., Darton, R., Netten, A., \& Lewis, A. (2013). The relationship between building design and residents' quality of life in extra care housing schemes. Health \& Place, 21, 52-64.

Pereira, A. S., Shitsuka, D. M., Parreira, F. J., \& Shitsuka, R. (2018). Metodologia da pesquisa científica. Ed. UAB/NTE/UFSM, Santa Maria/RS. 
Research, Society and Development, v. 10, n. 2, e8910212277, 2021

(CC BY 4.0) | ISSN 2525-3409 | DOI: http://dx.doi.org/10.33448/rsd-v10i2.12277

Ratcliffe, J., Lester, L. H., Couzner, L., \& Crotty, M. (2013). An assessment of the relationship between informal caring and quality of life in older community-dwelling adults-more positives than negatives?. Health \& social care in the community, 21(1), 35-46.

Souza, M. T. D., Silva, M. D. D., \& Carvalho, R. D. (2010). Revisão integrativa: o que é e como fazer. Einstein (São Paulo), 8(1), 102-106.

Tavares, D. M. S., Fachinelli, A. M. P., Dias, F. A., Bolina, A. F., \& de Paiva, M. M. (2015). Preditores da qualidade de vida de idosos urbanos e rurais. Revista Baiana de Enfermagem, 29(4), 361-371.

Teston, E. F., \& Marcon, S. S. (2014). Quality of life and living conditions from the viewpoint of residents in a seniors condominium. Revista Gaúcha de Enfermagem, 35(1), 124-130.

Teston, E. F., \& Marcon, S. S. (2015). Estudo comparativo da qualidade de vida dos idosos que vivem em condomínios contra moradores da comunidade. Investigación y Educación en Enfermería, 33(1), 53-62. 